\title{
A Case of Eccrine Porocarcinoma Accompanying Renal Cell Carcinoma
}

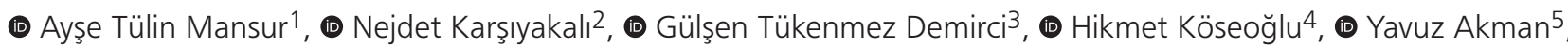 \\ (1) Şemsi Yıldız \\ ${ }^{1}$ Başkent University Faculty of Medicine, Department of Dermatology and Venereology, Istanbul, Turkey \\ 2 Acıbadem Mehmet Ali Aydınlar University, Altunizade Hospital, Clinic of Urology, Istanbul, Turkey \\ ${ }^{3}$ Acıbadem Mehmet Ali Aydınlar University, Altunizade Hospital, Clinic of Dermatology and Venereology, Istanbul, Turkey \\ 4 University of Health Sciences Turkey, Istanbul Training and Research Hospital, Clinic of Urology, Istanbul, Turkey \\ ${ }_{5}^{5}$ Memorial Hizmet Hospital, Clinic of Urology, Istanbul, Turkey \\ ${ }^{6}$ Başkent University Faculty of Medicine, Clinic of Pathology, Istanbul, Turkey
}

\begin{abstract}
In this case report, we present the coexistence of eccrine porocarcinoma (EPC) and renal cell carcinoma (RCC) in a 69-year-old male patient whose surgical resection pathology of the skin lesion in the right gluteal region was reported as EPC and concurrent radical nephrectomy pathology of the right renal mass was reported as RCC.

Keywords: Eccrine porocarcinoma, malignant eccrine poroma, poroid neoplasm, renal cell carcinoma, sweat gland tumour
\end{abstract}

\section{Introduction}

Eccrine porocarcinoma (EPC) is a rare, malignant skin adnexal tumour arising from the intraepidermal section of the eccrine sweat glands $(1,2)$. The aetiology of EPC is still unclear; it can be a de novo lesion or develop from eccrine poroma (EP). Chronic immunosuppression and ultraviolet light are etiologic factors $(1,2)$. EPC was firstly described by Pinkus and Mehregan (3) as a different entity in 1963. It is a solitary, pink-red nodule or plaque and typically seen in people aged $\geq 60$ years. Its estimated incidence is below $0.3 / 100,000$ in European countries and has comparable incidence in both genders (2). Local repeated recurrence and tumour metastasis are major clinical features of EPC, and the tumour tissue usually metastasise to local lymph nodes (LN) or skin and less common to the breast, liver, lungs, retroperitoneum and ovaries (4). In general, both local recurrence and LN metastasis can be seen in $20 \%$ of EPC cases, and the distant metastasis rate has been reported as $10 \%(5,6)$. Coexistence of EPC with haematologic malignancies, colorectal carcinoma and squamous cell carcinoma was reported separately $(7,8)$. However, coexistence of urological malignancies and EPC has not been reported yet.
Herein, we present the coexistence of EPC and renal cell carcinoma (RCC) in a 69-year-old male patient whose surgical resection pathology of skin lesion in the right gluteal region was reported as EPC and concurrent radical nephrectomy pathology of the right renal mass was reported as RCC.

\section{Case Presentation}

Written informed consent was obtained from patient for publication of his case and accompanying images.

A 69-year-old man presented with a swelling lesion in the right gluteal region that has been present approximately for 7 years and has grown and demonstrated ulceration for the last few months. He had no history of smoking, obesity or hypertension. A mass measuring $65 \times 60 \times 45 \mathrm{~mm}$ in the right kidney was detected by urinary system ultrasonography due to right side pain a month ago.

Dermatological examination revealed a pinkish grey oval plate of $25 \times 25 \mathrm{~mm}$ in diameter, with raised skin, crusts and superficial ulcers (Figure 1). Dermoscopic examination revealed pink, yellow, white and occasionally blue-grey asymmetrical areas and hairpin-shaped, linear and spot veins (Figure 2).

Cite this article as: Mansur AT, Karşıyakalı N, Tükenmez Demirci G, Köseoğlu H, Akman Y, Yıldız Ş. A Case of Eccrine Porocarcinoma Accompanying Renal Cell Carcinoma. Bull Urooncol 2021;20(3):186-191 


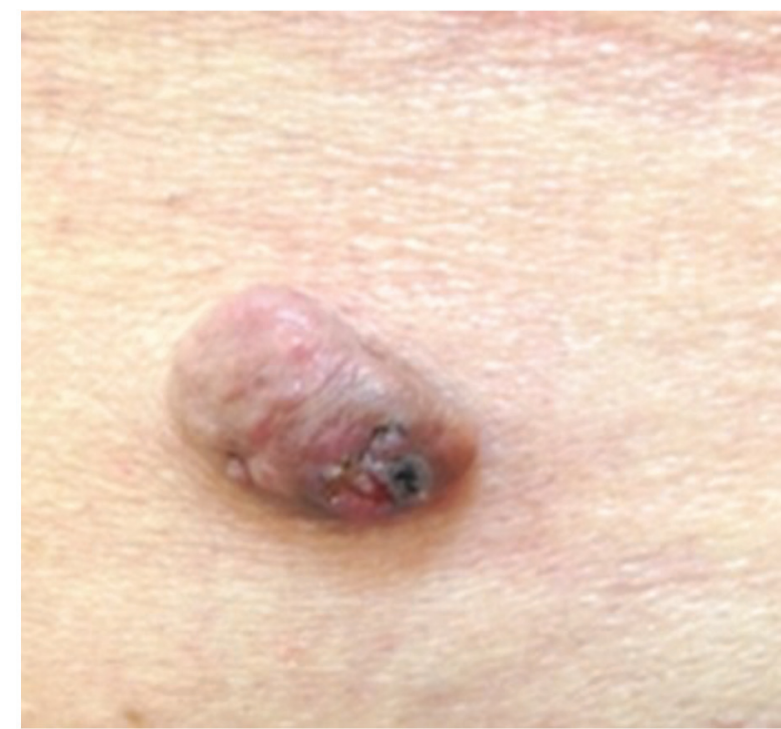

Figure 1. Appearance of the lesion: a pinkish grey oval plate with a diameter of $25 \times 25 \mathrm{~mm}$, with raised skin, crusts and superficial ulcers

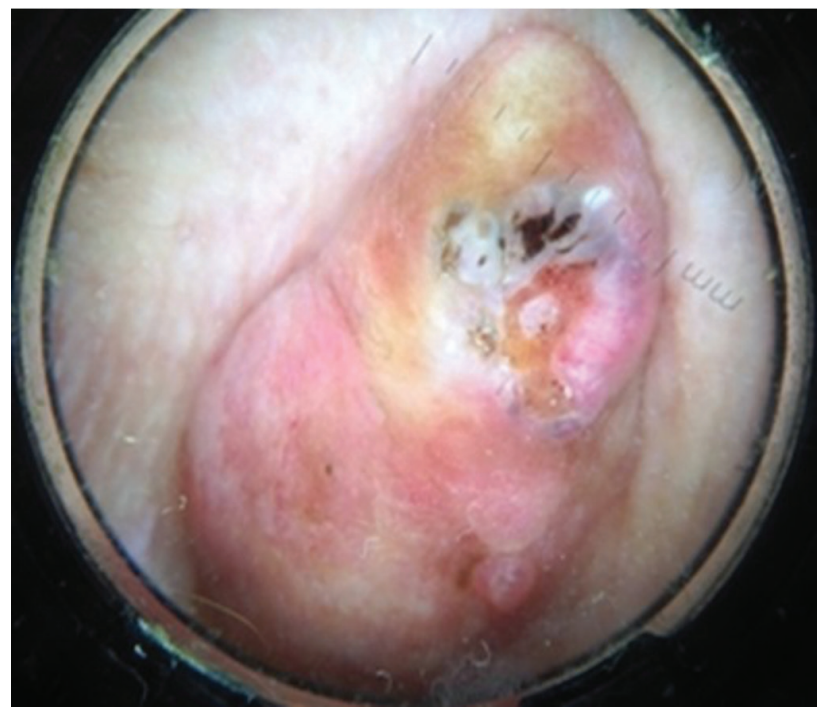

Figure 2. Dermoscopic appearance of the lesion

Surgical resection of the lesion revealed a solid tumour with desmoplastic stroma associated with the epidermis. Tumour cells forming large and small islands were malignant cells with large, pleomorphic vesicular nuclei, prominent nucleoli and large eosinophilic cytoplasm. Ductal and squamous differentiation areas, coagulation necrosis and calcification were observed (Figure $3 a$ and Figure $3 b$ ).

Immunohistochemical examination revealed that the cells forming the lesion were strongly positive with p63 and epithelial membrane antigen; by contrast, actin, carcinoembryonic antigen and cytokeratin (CK) 20 were negative. Ductal differentiation areas were positive for CK7 and luminal periodic acid Schiff, and squamous differentiation areas were positive for CK5. Ki-67 staining in the most intense area was 15\% (Figure $3 c$ and Figure $3 d)$.

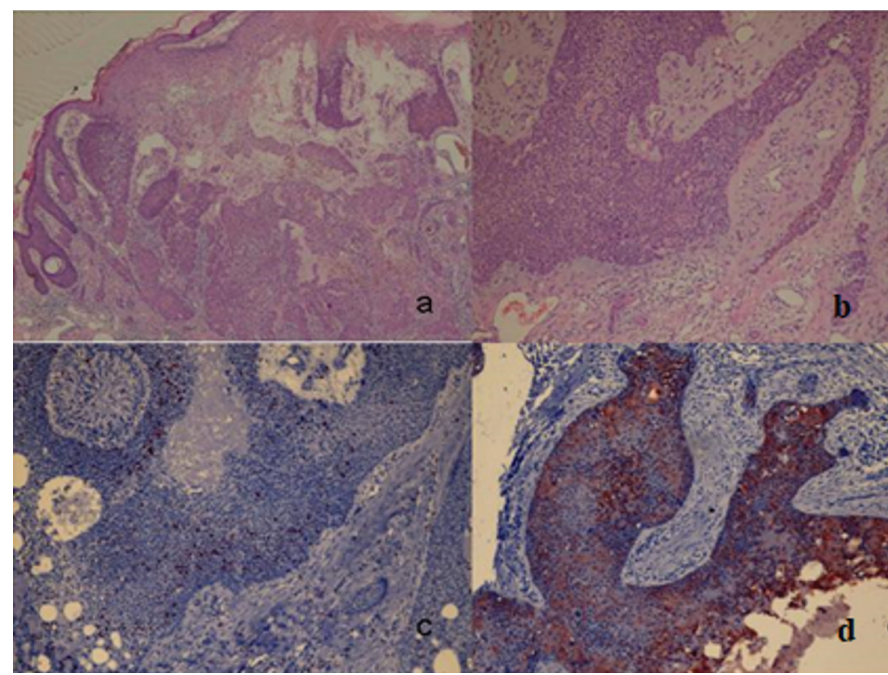

Figure 3. a. Neoplastic cells with invasion areas (hematoxylin and eosin (H\&E) staining, $\times 50)$, b. Neoplastic cells with large pleomorphic and hyperchromatic nuclei and islets of invasion at the periphery (H\&E, $\times 100)$, c. High Ki-67 ratio (Immunoperoxidase, $\times 100)$, d. Diffuse epithelial membrane antigen positivity (Immunoperoxidase, $\times 100$ )

The skin lesion was diagnosed as EPC according to clinical, histopathological and immunohistochemical findings.

No regional LN involvement or distant metastasis was detected in the contrast-enhanced thoraco-abdominal computed tomography, which was performed for clinical staging of the right renal mass. The patient underwent right laparoscopic radical nephrectomy, and histopathological examination of the renal mass revealed a stage 1 clear cell type RCC (pT1bN0M0). No local recurrence or distant metastasis of EPC or RCC was detected during the 2-year follow-up period.

\section{Discussion}

Coexistence of RCC, which is an important urological malignancy, and EPC, which is a rare dermatological malignancy, was found in a 69-year-old man. Although coexistence of EPC with various malignancies has been reported previously, to the best of our knowledge, no other case in the literature showed the coexistence of EPC and RCC.

Poroid neoplasms represent a spectrum of adnexal tumours arising from the intraepidermal section of the eccrine sweat glands. EP represents benign sections of these tumours, but if untreated for a long period, the risk of malignant transformation to EPC is definite (2). Well-defined risk factors for RCC include tobacco use, obesity and hypertension (9). However, these factors are not considered to increase the risk of developing an EPC. The aetiology of EPC is still unclear; it can be a de novo lesion or develop from EP. Chronic immunosuppression and ultraviolet light are aetiologic factors $(1,2)$. Bleeding, ulceration and growth are indicative of malignant transformation in an existing EP lesion $(2,10)$. Similarly, in the present case, the lesion in the right gluteal region grew and ulcerate during the last few months. In addition, an increase in vascularity in the lesion was detected by dermoscopic examination, as shown in Figure 2. 
EP is typically seen on the palms and soles, but at least $25 \%$ of the cases may occur in other areas such as the face and scalp. Although the typical clinical appearance is a pink-red, solitary nodule or plaque, pigmented variants were also described $(1,11,12)$. EPCs are frequently seen in the lower extremity, but can also be seen in the trunk, head, scalp and upper extremity (13). In the present case, the lesion was found in the right gluteal region, unusually.

A high mitotic activity, atypical mitosis and pleomorphic nucleus are strong indicators of EPC in histopathological examination (1). Similarly, histopathological examination revealed that the tumour cells had large eosinophilic cytoplasm, pleomorphic vesicular nucleus and prominent nucleoli. Remarkably, the lesion contained squamous differentiation, coagulation necrosis and calcification areas.

Tumours accompanying EPC include haematologic malignancies, colorectal carcinoma, tricholemmal carcinoma and squamous cell carcinoma $(7,8)$. Immunosuppression is considered the cause for the coexistence of EPC and haematologic malignancies (8). Dewan et al. (8) reported two patients who received immunosuppressive therapy for chronic lymphocytic leukaemia and red cell aplasia and polycythaemia rubra vera in two patients who died due to septicaemia and acute myeloid leukaemia, respectively (8).

\section{Conclusion}

EPC is a rare dermatological malignancy, typically seen in the older population. Although it is frequently observed in the lower extremities, it can also be seen in unusual areas such as the gluteal region. It is mostly seen as an isolated malignancy, but may accompany urological malignancies such as RCC.

\section{Acknowledgements}

Publication: The results of the study were not published in full or in part in form of abstracts.

Contribution: There is not any contributors who may not be listed as authors.

Conflict of Interest: No conflict of interest was declared by the authors.

Financial Disclosure: The authors declared that this study received no financial support.

\section{Ethics}

Informed Consent: Written informed consent was obtained from patient for publication of his case and accompanying images.
Peer-review: Externally peer-reviewed.

\section{Authorship Contributions}

Concept: A.T.M., G.T.D., Design: A.T.M., G.T.D., Data Collection or Processing: N.K., Ş.Y., Y.A., Analysis or Interpretation: N.K., G.T.D., Literature Search: N.K., Critical Review: H.K., A.T.M., Y.A., Writing: N.K., Ş.Y.

\section{References}

1. Robson A, Greene J, Ansari N, et al. Eccrine porocarcinoma (malignant eccrine poroma): a clinicopathologic study of 69 cases. Am J Surg Pathol 2001;25:710-720.

2. Sgouros D, Piana S, Argenziano G, et al. Clinical, dermoscopic and histopathological features of eccrine poroid neoplasms. Dermatology 2013;227:175-179.

3. Pinkus $\mathrm{H}$, Mehregan AH. Epidermotropic Eccrine Carcinoma. A Case Combining Features of Eccrine Poroma and Paget's Dermatosis. Arch Dermatol 1963;88:597-606.

4. de Bree E, Volalakis $E$, Tsetis $D$, et al. Treatment of advanced malignant eccrine poroma with locoregional chemotherapy. $\mathrm{Br}$ J Dermatol 2005;152:1051-1055.

5. Kalogeraki A, Tamiolakis D, Tsagatakis T, et al. Eccrine porocarcinoma: cytologic diagnosis by fine needle aspiration biopsy (FNAB). Acta Med Port 2013;26:467-470.

6. Marone U, Caraco C, Anniciello AM, et al. Metastatic eccrine porocarcinoma: report of a case and review of the literature. World J Surg Oncol 2011;9:32.

7. Cribier B, Lipsker D, Grosshans E. Eccrine porocarcinoma, tricholemmal carcinoma and multiple squamous cell carcinomas in a single patient. Eur J Dermatol 1999;9:483-486.

8. Dewan P, Moir G, Cerio R, et al. Aggressive eccrine porocarcinoma associated with haematological disorders: report of two cases. Clin Exp Dermatol 2010;35:e207-e209. doi: 10.1111/j.1365-2230.2009.03739.x.

9. Zhang GM, Zhu Y, Ye DW. Metabolic syndrome and renal cell carcinoma. World J Surg Oncol 2014;12:236.

10. Wen SY. Case report of eccrine porocarcinoma in situ associated with eccrine poroma on the forehead. J Dermatol 2012;39:649-651.

11. Nicolino R, Zalaudek I, Ferrara G, et al. Dermoscopy of eccrine poroma. Dermatology 2007;215:160-163.

12. Mitsuishi T, Ansai $S$, Ueno $T$, et al. Pigmented poroid neoplasm mimicking nodular melanoma. J Dermatol 2010;37:542-544.

13. Shiohara J, Koga $H$, Uhara $H$, et al. Eccrine porocarcinoma: clinical and pathological studies of 12 cases. J Dermatol 2007;34:516-522. 